\title{
SYSTEMS OF DIFFERENCE EQUATIONS WITH GENERAL HOMOGENEOUS BOUNDARY CONDITIONS $\left({ }^{1}\right)$
}

\author{
BY \\ STANLEY OSHER
}

1. Introduction. As Strang [2], [3] has pointed out, a natural tool for studying $l_{2}$ stability of difference equations which approximate hyperbolic or parabolic equations in one space variable is the Wiener-Hopf technique of factorization of Toeplitz matrices discussed in [4] by Gohberg and Krein.

Strang has discussed stability of difference equations whose solutions satisfy the special boundary condition $u=0$ on and outside of the boundaries. We shall use Toeplitz matrices to generalize this theory to systems of equations in one space variable with arbitrary homogeneous boundary conditions. The discussion will be confined to problems in the quarter plane with constant coefficients. The results can be easily generalized to variable coefficient and two point boundary value problems, using Kreiss' method [1] and/or Strang's in [2] and [3].

We shall derive Kreiss' sufficient conditions for stability of dissipative hyperbolic systems with constant coefficients as a corollary to a more general result. In particular, the condition of dissipativity is replaced by a weaker condition.

We treat the explicit case in the main part of this work and add the implicit case as an appendix in part 7. The main results are stated in XIX and XXVIII. Kreiss' Theorem is derived in XXII. We give nondissipative examples in XXIII and XXIX.

We hope to extend this technique to include problems in several space variables in the near future.

2. Basic problem. Consider a first order hyperbolic system of partial differential equations

(2.1) $u_{t}=A u_{x}$, where $A$ is a diagonal matrix with constant entries:

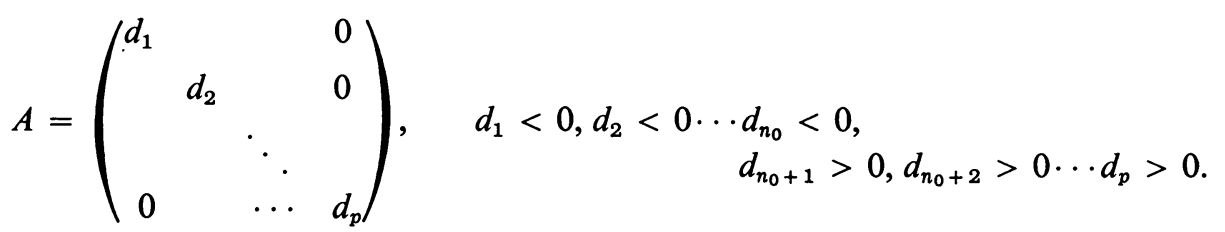

Take the initial-boundary value problem in the quarter plane

(2.2) $u(x, 0)=f(x) ; 0 \leqq x<\infty$,

Received by the editors January 21, 1968.

( ${ }^{1}$ ) This work performed under the auspices of the U.S. Atomic Energy Commission. 
(2.3) $u^{I}(0, t)=S_{I I} u^{I I}(0, t), S_{I I}$ is a rectangular constant $\left(n_{0} \times p-n_{0}\right)$ matrix.

$$
\begin{aligned}
u^{I} & =\left(u^{(1)}(x, t), \ldots, u^{\left(n_{0}\right)}(x, t)\right), \\
u^{I I} & =\left(u^{\left(n_{0}+1\right)}(x, t), \ldots, u^{(p)}(x, t)\right) .
\end{aligned}
$$

We approximate this system of partial differential equations by the system of difference equations

$$
v_{j}^{n+1}=\sum_{k=-s}^{l} C_{k} v_{j+k}^{n} ; \quad j=s, s+1, \ldots, n=0,1,2, \ldots
$$

with boundary conditions

(2.4) (b) $v_{j}^{n+1}=-\sum_{k=s}^{r+s} \alpha_{j+1, k+1} v_{k}^{n+1} ; \quad j=0,1, \ldots, s-1, n=0,1,2, \ldots$

$r, l$, and $s$ are nonnegative integers, $v_{j}^{n}$ is a $p$ component vector:

$$
v_{j}^{n}=\left[\begin{array}{c}
v_{j}^{n}(1) \\
v_{j}^{n}(2) \\
\vdots \\
v_{j}^{n}(p)
\end{array}\right] .
$$

The $C_{k}$ are constant diagonal $(p \times p)$ matrices. The $\alpha_{j k}$ are constant $(p \times p)$ matrices. (We shall sometimes write $\alpha_{j, k}$ for $\alpha_{j k}$.) $v_{j}^{0}=\Phi_{j}$ is a given arbitrary initial vector. For simplicity, we define $\alpha_{j k}=\delta(j-k) I$ if $1 \leqq j, k \leqq s$, and thus equation (b) may be rewritten

$(2.4)\left(\mathrm{b}^{\prime}\right) \quad \sum_{k=0}^{r+s} \alpha_{j+1, k+1} v_{k}^{n+1}=0, \quad n=0,1, \ldots, j=0,1, \ldots, s-1$.

Let $u(x, t)$ be the solution to (2.1) with initial conditions (2.2) and boundary conditions (2.3). We introduce a time step $k>0$ and a mesh-width $h>0$ and divide the interval $0 \leqq x<\infty$ into subintervals of length $h$. Assume $k / h=\mu=$ constant. Let

$$
\begin{aligned}
u(j h, n k) & =\hat{u}_{j}^{n}, & & j=0,1, \ldots, \\
f(j h) & =\hat{u}_{j}^{0}, & & n=0,1, \ldots
\end{aligned}
$$

We say the difference scheme is a consistent approximation to the differential equation if $\hat{u}_{j}^{n}$ satisfies 2.4(a) and 2.4(b) with a remainder which is of order $O\left(k^{2}\right)$ and $\Phi_{j}=f(j h)+O\left(h^{2}\right)$ for sufficiently smooth $u(x, t)$ and $f(x)$. Let us assume that the difference scheme is indeed consistent with the basic system of differential equations and boundary conditions.

$\left({ }^{2}\right)$ Notice that if $s=0$, the difference scheme is independent of the boundary conditions, and Strang's necessary and sufficient conditions [2] are valid. Thus, we shall take $s>0$ and $C_{-s} \neq[0]_{p \times p}$. 
We are interested in the convergence of this difference scheme, i.e. is it true that

$$
v_{j}^{n} \rightarrow u(j h, t)
$$

in some sense, as $k \rightarrow 0$ and $n k \rightarrow t \leqq T$, for some $T>0$ ? We make this question more precise with the help of the following definitions:

I. Definitions.

$$
\left(l_{2}\right)_{(p \times 1),(-\infty, \infty)}=\left\{f \mid\left(\sum_{j=-\infty}^{\infty}\left|f_{j}\right|^{2}\right)^{1 / 2}<\infty\right\}
$$

is a Hilbert space where each $f_{j}$ is a $p$ vector and

$$
\left|f_{j}\right|=\left(\sum_{k=1}^{p}\left|f_{j}(k)\right|^{2}\right)^{1 / 2} \text { and }\|f\|=\left(\sum_{j=-\infty}^{\infty}\left|f_{j}\right|^{2}\right)^{1 / 2} \text {. }
$$

$\left(\hat{l}_{2}\right)_{(p \times 1),(-\infty, \infty)}$ is the Fourier-transform space, i.e.

$$
\begin{aligned}
& \hat{f}\left(e^{i \theta}\right)=\sum_{j=-\infty}^{\infty} f_{j} e^{i j \theta} \\
& \|\hat{f}\|^{2}=\frac{1}{2 \pi} \int_{0}^{2 \pi}\left|\hat{f}\left(e^{i \theta}\right)\right|^{2} d \theta=\|f\|^{2} .
\end{aligned}
$$

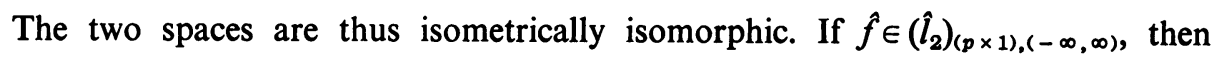
define

$$
P_{+} \hat{f}=\sum_{j=0}^{\infty} f_{j} e^{i j \theta} \quad \text { and } \quad P_{0} \hat{f}=f_{0}
$$

Define $P_{+}\left(\hat{l}_{2}\right)_{(p \times 1),(-\infty, \infty)}=\left(\hat{l}_{2}\right)_{(p \times 1)}$ and the definition of $\left(l_{2}\right)_{(p \times 1)}$ is obvious. In view of the isometric isomorphism, we shall often skip back and forth between the two spaces. We shall sometimes let $\hat{f}\left(e^{i \theta}\right)$ be extended to $\hat{f}(x)$ for $x$ off the unit circle and complex.

Throughout this work we shall assume that

$$
v^{n}=\left\{v_{0}^{n}, v_{1}^{n}, \ldots, v_{j}^{n}, \ldots\right\} \text { for } n=0,1, \ldots
$$

is an element in $\left(l_{2}\right)_{(p \times 1)}$. Hence equations (2.4a) and (2.4b) are to hold in this space. We also assume that

$$
\tilde{u}(t)=\{u(0, t), u(h, t), \ldots, u(j h, t), \ldots\} \text { for all } t,
$$

$0 \leqq t \leqq T$, is an element in $\left(l_{2}\right)_{(p \times 1)}$ (i.e. the original problem is well-posed). The question of convergence may be stated as follows. Is it true that

$$
\left\|\left(v^{n}-\tilde{u}(t)\right) h^{1 / 2}\right\| \rightarrow 0 \text { as } k \rightarrow 0 \text { and } n k \rightarrow t \leqq T ?
$$

For consistent difference schemes, we have the following well-known LaxRichtmyer equivalence theorem: Convergence of the difference scheme to the solution of the well-posed differential equation is equivalent to stability of the difference equations. 
II. Definition. The difference equations (2.4a) and (2.4b) are said to be stable if there exists a constant $M>0$ such that for any initial conditions $v_{j}^{0}=\Phi_{j}$ with $\Phi=\left\{\Phi_{0}, \Phi_{1}, \ldots\right\} \in\left(l_{2}\right)_{(p \times 1)}$ it follows that $\left\|v^{n}\right\| \leqq M\|\Phi\|$ for all $n \geqq 0$.

The basic problem of this work is in obtaining conditions on (2.4) which assure its stability.

We may obtain consistent boundary conditions many different ways. For example

$$
v_{0}^{n+1}=2 v_{s}^{n+1}-v_{2 s}^{n+1}
$$

is consistent with any differential equation or boundary condition as may be verified by merely examining the Taylor series

$$
\begin{aligned}
& 2 \hat{u}(s h, n k)=2\left[\hat{u}(0, n k)+\hat{u}_{x}(0, n k) s h+\hat{u}_{x x}\left(\theta_{1}, n k\right)(s h / 2)^{2}\right], \\
& \hat{u}(2 s h, n k)=\hat{u}(0, n k)+\hat{u}_{x}(0, n k) 2 s h+\hat{u}_{x x}\left(\theta_{2}, n k\right)(2 s h / 2)^{2} .
\end{aligned}
$$

Consistency follows because $k / h=$ constant. Clearly, we do not expect such trivial boundary conditions to be appropriate regardless of the underlying partial differential equation. We shall discuss in another paper the question of appropriate boundary conditions

III. REMARK. The mapping of $v^{n}$ to $v^{n+1}$ defined by the equations (2.4) is clearly linear. That is, there exists a linear operator $L$ such that $v^{n+1}=L v^{n}$. This operator $L$ may be written as a finite-dimensional perturbation of a convolution operator premultiplied by an orthogonal projection.

More precisely, we define

and recall

$$
C(x)=\sum_{k=-s}^{l} C_{k} x^{-k}
$$

$$
\begin{aligned}
P_{+} \sum_{j=-\infty}^{\infty} f_{j} x^{j}=\sum_{j=0}^{\infty} f_{j} x^{j}, & P_{0} \sum_{j=0}^{\infty} f_{j} x^{j}=f_{0}, \\
\alpha_{j k}=\delta(j-k) I & \text { if } 1 \leqq j, k \leqq s .
\end{aligned}
$$

Then we may write $L=T+S$, where $T u(x)=P_{+} C(x) u(x)$ defines the Toeplitz operator, and

$$
S u(x)=-\sum_{j=0}^{s-1} x^{j} P_{0}\left(\sum_{k=0}^{r+s} x^{-k} \alpha_{j+1, k+1} C(x) u(x)\right)
$$

defines the finite-dimensional operator. Thus we may restate the basic problem. Obtain conditions on the $C_{k}$ and the $\alpha_{j k}$ such that $\exists$ a constant $M>0$ with $\left\|(T+S)^{n}\right\|$ $\leqq M$ for all $n \geqq 0$.

In what follows, we shall treat the question of stability independently of consistency, unless otherwise stated. Thus, we shall concern ourselves with powerboundedness of $T+S$ and forget about the differential equations until we explicitly mention them. 
3. Preliminary stability theorem (Kreiss). We need an important stability theorem which is a generalization of a result of H.-O. Kreiss [1].

IV. Lemma. Let L be a bounded linear operator on a Hilbert space $H$ and $P$ be an orthogonal projection on $H$ such that $\|(I-P) L\| \leqq 1$.

Then for any $\Phi \in H$ :

$$
\left\|L^{n} \Phi\right\|^{2} \leqq\|\Phi\|^{2}+\sum_{k=1}^{n}\left\|P L^{k} \Phi\right\|^{2}
$$

Proof.

$$
\begin{aligned}
\|L \Phi\|^{2} & =\|P L \Phi\|^{2}+\|(I-P) L \Phi\|^{2} \\
& \leqq\|P L \Phi\|^{2}+\|\Phi\|^{2} \text { because }\|(I-P) L\| \leqq 1 .
\end{aligned}
$$

Suppose the result is valid for $L^{k} \Phi, k=1,2, \ldots, n$.

$$
\begin{aligned}
\left\|L^{n+1} \Phi\right\|^{2} & =\left\|P L^{n+1} \Phi\right\|^{2}+\left\|(I-P) L L^{n} \Phi\right\|^{2} \\
& \leqq\left\|P L^{n+1} \Phi\right\|^{2}+\left\|L^{n} \Phi\right\|^{2} \\
& \leqq\left\|P L^{n+1} \Phi\right\|^{2}+\sum_{k=1}^{n}\left\|P L^{k} \Phi\right\|^{2}+\|\Phi\|^{2} .
\end{aligned}
$$

V. Lemma. Let $L$ be a bounded linear operator on $H$ and $P$ be an orthogonal projection on $H$ such that $\|L(I-P)\| \leqq 1$.

Then, for any $\Phi \in H$ :

$$
\sum_{j=0}^{\infty}\left\|P(L(I-P))^{j} \Phi\right\|^{2} \leqq\|\Phi\|^{2} .
$$

Proof. We shall show that

$$
\sum_{j=0}^{n-1}\left\|P(L(I-P))^{j} \Phi\right\|^{2}+\left\|(L(I-P))^{n} \Phi\right\|^{2} \leqq\|\Phi\|^{2}
$$

by induction on $n$. For $n=1$

$$
\|P \Phi\|^{2}+\|(I-P) \Phi\|^{2}=\|\Phi\|^{2}
$$

and $\|L(I-P) \Phi\|=\|L(I-P)(I-P) \Phi\| \leqq\|(I-P) \Phi\|$ because $(I-P)$ is idempotent and $\|L(I-P)\| \leqq 1$. Thus

$$
\|P \Phi\|^{2}+\|L(I-P) \Phi\|^{2} \leqq\|\Phi\|^{2} .
$$

The induction step is similar with $(L(I-P))^{n} \Phi$ used in place of $\Phi$.

VI. Theorem. Let $L$ be a bounded linear operator on a Hilbert space H. Let $P$ be an orthogonal projection on $H$. Assume

(1) $\|(I-P) L\| \leqq 1$,

(2) $\|L(I-P)\| \leqq 1$. 
(3) There exists a sequence of nonnegative numbers $\left\{a_{n}\right\}$ such that $\sum_{n=0}^{\infty} a_{n}=a<\infty$ and $\left\|P L^{n} P\right\| \leqq a_{n}$ for all $n=0,1, \ldots$

Then $\left\|L^{n}\right\| \leqq\left(1+a^{2}\right)^{1 / 2}$ for all $n=0,1, \ldots$

Proof. Consider the following well-known identity for any pair of operators $A$ and $B$.

$$
A^{n}=B^{n}+\sum_{j=1}^{n} A^{j-1}(A-B) B^{n-j}
$$

Choose $A=L, B=L(I-P)$. We thus have the identity:

$$
L^{n}=(L(I-P))^{n}+\sum_{j=1}^{n} L^{j} P(L(I-P))^{n-j} .
$$

It then follows by the triangle inequality, the idempotence of $P$, and hypothesis (3) that:

$$
\begin{aligned}
\left\|P L^{n} \Phi\right\| & \leqq \sum_{j=0}^{n}\left\|P L^{j} P P(L(I-P))^{n-j} \Phi\right\| \\
& \leqq \sum_{j=0}^{n} a_{j}\left\|P(L(I-P))^{n-j} \Phi\right\| .
\end{aligned}
$$

By the Schwarz inequality:

$$
\begin{aligned}
\left\|P L^{n} \Phi\right\|^{2} & \leqq \sum_{j=0}^{n} a_{j} \sum_{t=0}^{n} a_{t}\left\|P(L(I-P))^{n-t} \Phi\right\|^{2} \\
& \leqq a \sum_{t=0}^{n} a_{\imath}\left\|P(L(I-P))^{n-t} \Phi\right\|^{2} \\
\sum_{n=0}^{\infty}\left\|P L^{n} \Phi\right\|^{2} & \leqq a \sum_{t=0}^{\infty} a_{t} \sum_{n=t}^{\infty}\left\|P(L(I-P))^{n-t} \Phi\right\|^{2} \leqq a^{2}\|\Phi\|^{2},
\end{aligned}
$$

using Lemma V. By Lemma IV and the last inequality:

$$
\left\|L^{n} \Phi\right\|^{2} \leqq\|\Phi\|^{2}+\sum_{j=1}^{n}\left\|P L^{j} \Phi\right\|^{2} \leqq\|\Phi\|^{2}+a^{2}\|\Phi\|^{2}
$$

or $\left\|L^{n}\right\|^{2} \leqq 1+a^{2}$ for each $n=0,1, \ldots$ Q.E.D.

4. Necessary conditions for stability (Ryabenkii-Godunov).

VII. REMARK. If an operator $L$ is power-bounded, it is necessary that its spectral radius lie inside the unit circle: $|\sigma(L)| \leqq 1$.

For the operator $T+S$ defined in III, we shall show that if its spectral radius lies inside the unit circle, then

$$
\sup _{0 \leqq \theta \leqq 2 \pi ; \nu=1,2, \ldots, p}\left|c^{(v)}\left(e^{i \theta}\right)\right| \leqq 1
$$


where

$$
C(x)=\left[\begin{array}{ccccc}
c^{(1)}(x) & & 0 & & 0 \\
& \ddots & & & \\
0 & & c^{(v)}(x) & & 0 \\
& & & \ddots & \\
0 & & 0 & & c^{(p)}(x)
\end{array}\right]
$$

Suppose we assume $c^{\left(v_{0}\right)}\left(e^{i \theta_{0}}\right)=\lambda_{0}$ and $\left|\lambda_{0}\right|>1$. Let $v_{N}(j) \equiv 0$ if $j \neq \nu_{0}$,

$$
v_{N}\left(\nu_{0}\right)=\left\{0,0, \ldots, 0, \frac{e^{-i(r+s+l+1) \theta_{0}}}{\sqrt{ } N}, \ldots, \frac{e^{-i(r+s+l+N) \theta_{0}}}{\sqrt{ } N}, 0, \ldots\right\} .
$$

(The first nonvanishing term above is in the $r+s+l+1$ place.) Then $\left\|v_{N}\right\| \equiv 1$, yet $\left\|\left(T+S-\lambda_{0}\right) v_{N}\right\| \rightarrow 0$ as $N \rightarrow \infty$.

$$
\begin{aligned}
\therefore & \lambda_{0} \text { is in the spectrum of } T+S . \\
& \lambda_{0} \in \sigma(T+S) \text { and }\left|\lambda_{0}\right|>1 .
\end{aligned}
$$

Moreover, it follows easily that

$$
\|T\| \leqq 1 \Leftrightarrow \sup _{v=1,2, \ldots, p ; 0 \leqq \theta \leqq 2 \pi}\left|c^{(v)}\left(e^{i \theta}\right)\right| \leqq 1 .
$$

Consider $T-\lambda+S$ where $\lambda$ is a complex number and $\|T\| \leqq 1<|\lambda|$. It is clear that $T-\lambda$ is invertible. It is a well-known theorem e.g. [11] that if $R$ is invertible and $S$ is finite dimensional, then $R+S$ is invertible iff $R+S$ annihilates no nonzero vector.

We now have the following results: (a) $\Rightarrow$ (b) $\Leftrightarrow$ (c).

(a) $T+S$ is power-bounded.

(b) $|\sigma(T+S)| \leqq 1$.

(c) $\|T\| \leqq 1$ and, if $|\lambda|>1$, then $(T+S-\lambda) u=0 \Leftrightarrow u=0$

$$
\left(\|T\| \leqq 1 \Leftrightarrow \sup _{v=1,2, \ldots, p ; 0 \leqq \theta \leqq 2 \pi}\left|c^{(v)}\left(e^{i \theta}\right)\right| \leqq 1\right) .
$$

It is not true in general that (c) $\Rightarrow$ (a). For example consider the scalar difference equation:

$$
v_{j}^{n+1}=v_{j-1}^{n}, \quad j=1,2, \ldots, n=0,1, \ldots
$$

with boundary conditions

$$
v_{0}^{n+1}=2 v_{1}^{n+1}-v_{2}^{n+1}, \quad n=0,1, \ldots
$$

This is consistent with $u_{t}=-u_{x}(0 \leqq t, x)$ for $k / h=1$. $C\left(e^{i \theta}\right)=e^{i \theta}$; hence $\|T\| \leqq 1$.

If $\left(T+S-\lambda_{0}\right) \sum_{j=0}^{\infty} \Psi_{j} x^{j}=0$, then $\lambda_{0} \Psi_{j}=\Psi_{j-1}, j=1,2, \ldots$ Thus $\Psi_{j}=d / \lambda_{0}^{j}$ for some constant $d$ and

$$
d\left[1-2 / \lambda_{0}+1 / \lambda_{0}^{2}\right]=0 \Leftrightarrow d=0 \text { and } / \text { or } \lambda_{0}=1 .
$$


Thus condition (c) is verified. However, if we let $v_{j}^{0} \equiv 0$ if $j>0$ and $v_{0}^{0}=1$, then $v_{j}^{n}=\max (n-j+1,0)$ and we, of course, have instability. We thus need additional conditions to assure power-boundedness of $T+S$.

The condition that $\|T\| \leqq 1$ is just the well-known von Neumann criterion which is necessary for stability of pure initial value problems. The condition that $T+S$ should have no eigenvalues outside the unit circle is merely the additional Ryabenkiî-Godunov condition which is needed for the mixed initial-boundary problem, (see [12]).

In general, if $|\sigma(L)| \leqq 1$, then $\forall \varepsilon>0 \exists k(\varepsilon)>0$ such that $\left\|L^{n}\right\| \leqq k(\varepsilon)(1+\varepsilon)^{n}$.

We can show a stronger result for our difference operator $T+S$ :

If $|\sigma(T+S)| \leqq 1$, then $\exists k_{1}>0$ and $k_{2}>0$ such that

$$
\left\|(T+S)^{n}\right\| \leqq k_{1} n^{k_{2}} .
$$

We shall omit the proof of this result.

\section{Resolvent of $T+S$.}

VIII. Lemma. Let

(1) $A$ be an invertible operator,

(2) $B$ be finite dimensional,

(3) $(A+B) u=0 \Leftrightarrow u=0$.

Then $(A+B)^{-1}$ exists and

$$
(A+B)^{-1}=A^{-1}-A^{-1}\left(I+B A^{-1}\right)^{-1} B A^{-1} .
$$

Proof. We may show $A+B$ is invertible by using the same well-known theorem that we used in VII for $R+S$. We have the general identities for $A$ and $B$ in a noncommutative algebra, $A$ and $A+B$ both invertible.

$$
\begin{aligned}
(A+B)^{-1}(A+B) & =I \\
(A+B)^{-1}\left(I+B A^{-1}\right) & =A^{-1} \\
(A+B)^{-1} & =A^{-1}-(A+B)^{-1} B A^{-1} \\
& =A^{-1}-A^{-1}\left(I+B A^{-1}\right)^{-1} B A^{-1}
\end{aligned}
$$

IX. Corollary. If

(1) $\sup _{0 \leqq \theta \leqq 2 \pi ; v=1,2, \ldots, p}\left|c^{(v)}\left(e^{i \theta}\right)\right| \leqq 1 \Leftrightarrow\|T\| \leqq 1$,

(2) $(T+S-\lambda) \psi=0$ for $|\lambda|>1 \Rightarrow, \psi=0$.

Then $(T+S-\lambda)^{-1}$ exists and

$$
(T+S-\lambda)^{-1}=(T-\lambda)^{-1}-(T-\lambda)^{-1}\left(I+S(T-\lambda)^{-1}\right)^{-1} S(T-\lambda)^{-1} .
$$

Proof. In the last lemma, replace $A$ by $T-\lambda$ and $B$ by $S$.

X. Lemma AND Definition. Suppose $A$ is invertible and $B$ is finite dimensional. Then $A+B$ fails to be invertible iff there exists a vector $v$ in the range of $B$ such that $v=-B A^{-1} v$.

Let $\|T\| \leqq 1<|\lambda|$. Then, if we let $D(\lambda)$ be the finite-dimensional operator mapping 
the range of $S$ into itself:

$$
D(\lambda) P_{S}=P_{S}+S(T-\lambda)^{-1} P_{S},
$$

where $P_{S}$ is the orthogonal projection onto the range of $S$, it follows that

$$
(T+S-\lambda) u=0 \Rightarrow u=0 \Leftrightarrow|D(\lambda)| \neq 0 .
$$

(We write $|F|$ for the determinant of the matrix $F$.)

Proof. $A+B$ fails to be invertible iff there exists $u \neq 0$ such that $(A+B) u=0$. Let $A u=v \neq 0$. Hence $\left(I+B A^{-1}\right) v=0$ or $v=-B A^{-1} v$.

The second result follows if we substitute $S$ for $B$ and $T-\lambda$ for $A$.

XI. Remark. We now notice that the expression (5.1) for the resolvent of $T+S$ may be rewritten

$$
(T+S-\lambda)^{-1}=(T-\lambda)^{-1}-(T-\lambda)^{-1}[D(\lambda)]^{-1} S(T-\lambda)^{-1}
$$

We shall obtain $(T-\lambda)^{-1}$ using the theory of Toeplitz matrices. We then shall use this resolvent with (5.2) to obtain a matrix representation for the finite dimensional operator $D(\lambda)$. This gives us a simple expression for $(T+S-\lambda)^{-1}$. We shall use the resolvent of $T+S$ as a generating function for the powers of $T+S$, and thus, with the help of Theorem VI, we shall obtain the stability conditions.

The following discussion of Toeplitz matrices contains only those elements of the theory necessary for our work here.

XII. Lemma AND Definition. Let

$$
b(x)=b_{-s} x^{s}+\cdots+b_{l} x^{-l}, \quad s \geqq 0 \geqq-l, b_{-s} \neq 0 \neq b_{l} .
$$

Then the Toeplitz operator on $\left(\hat{l}_{2}\right)_{(1 \times 1)}$ defined by

$$
T_{b} f=P_{+} b\left(e^{i \theta}\right) \hat{f}\left(e^{i \theta}\right)
$$

is a bounded linear operator with norm

$$
\left\|T_{b}\right\| \leqq \sup _{0 \leqq \theta \leqq 2 \pi}\left|b\left(e^{i \theta}\right)\right| .
$$

Moreover, suppose

(1) $b\left(e^{i \theta}\right) \neq 0, \quad 0 \leqq \theta \leqq 2 \pi$ and

(2) index $0 \leqq \theta \leqq 2 \pi b\left(e^{i \theta}\right)=0$.

It then follows that

$\left(1^{\prime}\right) b(x)$ has the unique factorization $b(x)=b_{-}(x) b_{+}(x)$, where

$$
\begin{gathered}
b_{-}(x)=\prod_{i=1}^{l}\left(1-\frac{y_{i}}{x}\right), \quad\left|y_{i}\right|<1, i=1,2, \ldots, l, \\
b_{+}(x)=b_{-s} \prod_{i=1}^{s}\left(x-x_{i}\right), \quad\left|x_{i}\right|>1, i=1,2, \ldots, s ; \\
\left(\prod_{i=a}^{b} k_{i} \equiv 1 \text { if } b<a\right),
\end{gathered}
$$

and hence $b_{-}(x)$ is analytic and nontanishing for $|x| \geqq 1 ; b_{+}(x)$ is analytic and nonvanishing for $|x| \leqq 1$. 
(2') $T_{b}$ is invertible and

$$
T_{b}^{-1} u=P_{+} b_{+}^{-1} P_{+} b_{-}^{-1} u .
$$

Proof. By the Fundamental Theorem of Algebra, there exist $l+s$ constants which we denote by $\left\{x_{i}\right\}, i=1,2, \ldots, s,\left\{y_{i}\right\}, i=1, \ldots, l$; such that

$$
b(x) x^{l}=b_{-s} \prod_{i=1}^{s}\left(x-x_{i}\right) \prod_{i=1}^{l}\left(x-y_{i}\right)
$$

or

$$
b(x)=b_{-s} \prod_{i=1}^{s}\left(x-x_{i}\right) \prod_{i=1}^{l}\left(1-\frac{y_{i}}{x}\right) .
$$

Conditions (1) and (2) and the Argument Principle assure us that the number of solutions to $b(x)=0$ inside the unit circle is equal to the number of poles of $b(x)$ at $x=0$ which is $l$. Similarly, the number of solutions to $b(x)=0$ outside the unit circle is equal to the number of poles of $b(x)$ at $x=\infty$, which is $s$. Thus, we may choose $x_{1}, x_{2}, \ldots, x_{s}$ such that each $\left|x_{i}\right|>1$ and $y_{1}, y_{2}, \ldots, y_{l}$ such that each $\left|y_{j}\right|<1$.

Thus $b(x)=b_{-}(x) b_{+}(x)$.

Each of the functions: $b(x), b_{-}^{-1}(x), b_{+}^{-1}(x)$ is analytic in some neighborhood of the unit circle. Let $g(x)$ be any such function. Then, for any $\hat{f}\left(e^{i \theta}\right) \in\left(\hat{l}_{2}\right)_{(1 \times 1)}$

$$
\begin{aligned}
\left\|P_{+} g \hat{f}\right\| & \leqq\|g \hat{f}\|=\left(1 / 2 \pi \int_{0}^{2 \pi}\left|g\left(e^{i \theta}\right) \hat{f}\left(e^{i \theta}\right)\right|^{2} d \theta\right)^{1 / 2} \\
& \leqq \sup _{0 \leqq \theta \leqq 2 \pi}\left|g\left(e^{i \theta}\right)\right|\|\hat{f}\| .
\end{aligned}
$$

It is thus clear that $P_{+} b\left(e^{i \theta}\right) u\left(e^{i \theta}\right), P_{+} b_{-}^{-1}\left(e^{i \theta}\right) u\left(e^{i \theta}\right)$, and $P_{+} b_{+}^{-1}\left(e^{i \theta}\right) u\left(e^{i \theta}\right)$ define bounded linear operators on $\left(\hat{l}_{2}\right)_{(1 \times 1)}$, and

$$
\left\|T_{b}\right\| \leqq \sup _{0 \leqq \theta \leqq 2 \pi}\left|b\left(e^{i \theta}\right)\right| \text {. }
$$

Suppose we wish to solve $P_{+} b\left(e^{i \theta}\right) u\left(e^{i \theta}\right)=v\left(e^{i \theta}\right)$. We have

$$
b\left(e^{i \theta}\right) u\left(e^{i \theta}\right)=v\left(e^{i \theta}\right)+k_{-}\left(e^{i \theta}\right)
$$

where $k_{-}\left(e^{i \theta}\right)=\sum_{j=-\infty}^{-1} k_{j} e^{+i j \theta}$ is some unknown function. Then

$$
b_{-} b_{+} u=v+k_{-}, \quad b_{+} u=b_{-}^{-1} v+b_{-}^{-1} k_{-},
$$

but $P_{+} b_{-}^{-1} k_{-}=0$;

$$
\therefore b_{+} u=P_{+} b_{+} u=P_{+} b_{-}^{-1} v \text { or } u=b_{+}^{-1} P_{+} b_{-}^{-1} v \text {. }
$$

Moreover

$$
P_{+} b b_{+}^{-1} P_{+} b_{-}^{-1} v=P_{+} b_{-} P_{+} b_{-}^{-1} v=P_{+} v+P_{+} b_{-}\left(I-P_{+}\right) b_{-}^{-1} v
$$

but $P_{+} b_{-}\left(I-P_{+}\right)=0$;

$$
\therefore T_{b}^{-1} u=b_{+}^{-1} P b_{-}^{-1} u \text {. }
$$

XIII. Remark. The operator $T-\lambda$ may be written as $T_{(C-\lambda)}$ on the space $\left(l_{2}\right)_{(p \times 1)}$. The question of inverting it thus becomes one of factoring each function $c^{(v)}(x)-\lambda$ when $|\lambda|>1$. 
XIV. Lemma. Let

$$
p(x)=\sum_{j=-s}^{l} p_{j} x^{-s}=p_{-s} x^{s}+\cdots+p_{l} / x^{l}
$$

for $s \geqq-l$ have the property that

$$
\sup _{0 \leqq \theta \leqq 2 \pi}\left|p\left(e^{i \theta}\right)\right| \leqq 1 .
$$

Let $\lambda$ be a complex number such that $|\lambda|>1$. Let $p_{-s} \neq 0 \neq p_{l}$. Then

(1) $p\left(e^{i \theta}\right)-\lambda \neq 0,0 \leqq \theta \leqq 2 \pi$ and

(2) index $_{0 \leqq \theta \leqq 2 \pi}\left[p\left(e^{i \theta}\right)-\lambda\right]=0$.

It then follows that

$$
p(x)-\lambda=q(\lambda) \prod_{i=1}^{s}\left(x-x_{i}(\lambda)\right) \prod_{i=1}^{l}\left(1-\frac{y_{.}(\lambda)}{x}\right) .
$$

Each $\left|x_{i}(\lambda)\right|>1$, each $\left|y_{i}(\lambda)\right|<1$, and $q(\lambda)$ is a nonzero number which may depend on $\lambda$.

Proof. Since $\left|p\left(e^{i \theta}\right)\right|<|\lambda|$, conditions (1) and (2) are immediate. We then apply Lemma XII to $b(x)=p(x)-\lambda$; the result then follows.

XV. Definition and Corollary. Let

$$
\sup _{0<\theta \leqq 2 \pi ; \nu=1,2, \ldots, p}\left|c^{(v)}\left(e^{i \theta}\right)\right| \leqq 1 .
$$

Let $\lambda$ be a complex number with $|\lambda|>1$. Then, by the last lemma, we may factor

$$
c^{(\nu)}(x)-\lambda=d_{v}(\lambda) \prod_{i=1}^{s_{v}}\left(x-x_{i}^{(\nu)}(\lambda)\right) \prod_{i=1}^{l_{v}}\left(1-\frac{y_{i}^{(\nu)}(\lambda)}{x}\right) .
$$

Each $\left|x_{i}^{(v)}(\lambda)\right|>1, i=1,2, \ldots, s_{v} ;$ each $\left|y_{i}^{(v)}(\lambda)\right|<1, i=1,2, \ldots, l_{v}$,

$$
\begin{gathered}
c^{(v)}(x)=c_{-s_{v}}^{(v)} x^{s_{v}}+\cdots+c_{l_{v}}^{(v)} / x^{l_{v}}, \quad s_{v} \geqq-l_{v}, \\
c_{-s_{v}}^{(v)} \neq 0 \neq c_{l_{v}}^{(v)} .
\end{gathered}
$$

The number $d_{v}(\lambda)$ is a nonzero constant which may depend on $\lambda$. Let

$$
\begin{aligned}
& A_{-}(x, \lambda)=\left[\begin{array}{cccc}
\prod_{i=1}^{l_{1}}\left(1-\frac{y_{i}^{(1)}(\lambda)}{x}\right) & 0 & \ldots & 0 \\
0 & & 0 \\
0 & & \prod_{i=1}^{l_{p}}\left(1-\frac{y_{i}^{(p)}(\lambda)}{x}\right)
\end{array}\right] \text {, } \\
& A_{+}(x, \lambda)=\left[\begin{array}{ccc}
d_{1}(\lambda) \prod_{i=1}^{s_{1}}\left(x-x_{i}^{(1)}(\lambda)\right) & \cdots & 0 \\
0 & \cdots & d_{p}(\lambda) \prod_{i=1}^{s_{p}}\left(x-x_{i}^{(p)}(\lambda)\right)
\end{array}\right] \text {. }
\end{aligned}
$$


Then

$$
C\left(e^{i \theta}\right)-\lambda=A_{-}\left(e^{i \theta}, \lambda\right) A_{+}\left(e^{i \theta}, \lambda\right) .
$$

Thus, we may state as our main conclusion of this section:

$$
(T-\lambda)^{-1} u(x)=A_{+}^{-1}(x, \lambda) P_{+} A_{-}^{-1}(x, \lambda) u(x) .
$$

Proof. This result follows in a straightforward way from the results of the last three sections.

We have thus obtained the resolvent of $T$. We shall now obtain the matrix $D(\lambda) P_{S}$ defined in (5.2) and then use (5.3) to get the resolvent of $T+S$.

XVI. Definition AND Lemma. Let $\sup \left|c^{(v)}\left(e^{i \theta}\right)\right| \leqq 1$. Let $\lambda$ be a complex number with $|\lambda|>1$.

$A_{+}^{-1}(x, \lambda)$ and $A_{-}^{-1}(x, \lambda)$ have expansions

$$
\begin{array}{ll}
A_{+}^{-1}(x, \lambda)=\sum_{j=0}^{\infty} A_{+(j)}^{(-1)}(\lambda) x^{j} & \text { convergent for }|x|<1 . \\
A_{-}^{-1}(x, \lambda)=\sum_{j=0}^{\infty} A_{-(j)}^{(-1)}(\lambda) x^{-j} & \text { convergent for }|x|>1 .
\end{array}
$$

Let $H(\lambda)$ be the upper triangular matrix

$$
H(\lambda)=\left[\begin{array}{cccc}
{\left[A_{-(0)}^{(-1)}\right.} & \cdots & & {\left[A_{-(s-1)}^{(-1)}\right.} \\
{[0]} & {\left[A_{-(0)}^{(-1)}\right]} & \cdots & {\left[A_{-(s-2)}^{(-1)}\right]} \\
\vdots & & & \\
{[0]} & & & {\left[A_{-(0)}^{(-1)}\right]}
\end{array}\right]_{((s p) \times(s p))}
$$

Let $\hat{\alpha}$ be the matrix of boundary conditions

$$
\hat{\alpha}=\left[\begin{array}{ccc}
{\left[\alpha_{11}\right]\left[\alpha_{12}\right]} & \cdots & {\left[\alpha_{1, r+s+1}\right]} \\
{\left[\alpha_{21}\right]\left[\alpha_{22}\right]} & \cdots & \vdots \\
{\left[\alpha_{s, 1}\right]\left[\alpha_{s, 2}\right]} & \cdots & {\left[\alpha_{s, r+s+1}\right]}
\end{array}\right]_{(s p) \times((r+s+1) p)}
$$

Finally consider $\mathscr{A}(\lambda)$, which is a matrix consisting of the first $(r+s+1) p$ components of the $s p$ linearly independent solutions to the problem

$$
\sum_{k=-s}^{l} C_{k} v_{k+j}=\lambda v_{j}, \quad j=s, s+1, \ldots
$$

This matrix is

$$
\mathscr{A}(\lambda)=\left[\begin{array}{cccc}
{\left[A_{+(0)}^{(-1)}\right]} & {[0]} & \cdots & {[0]} \\
{\left[A_{+(1)}^{(-1)}\right]} & {\left[A_{+(0)}^{(-1)}\right]} & \cdots & {[0]} \\
& & \ddots & {\left[A_{+(0)}^{(-1)}\right]} \\
{\left[A_{+(r+s)}^{(-1)}\right]} & {\left[A_{+(r+s+1)}^{(-1)}\right]} & & {\left[A_{+(r+1)}^{(-1)}\right]}
\end{array}\right](((r+s+1) p) \times(s p))
$$


Now we may state our main results of this section:

$$
D(\lambda) P_{S}=-\lambda F(\lambda) H(\lambda) P_{s}
$$

where

$$
F(\lambda)=\hat{\alpha} \mathscr{A}(\lambda) .
$$

Moreover $|H(\lambda)| \equiv 1$, and $\left|D\left(\lambda_{0}\right)\right|=0$ iff $\left|F\left(\lambda_{0}\right)\right|=0$.

Proof. The first result is just a straightforward calculation. $D(\lambda)$ is the matrix defined on the range of $S$ by the operator

$$
\begin{gathered}
P_{S}+S(T-\lambda)^{-1} P_{S}=D(\lambda) P_{S} . \\
P_{S} f=\sum_{j=0}^{s-1} g_{j} x^{j} \quad \text { for some vectors } g_{j}(f) . \\
S u=-\sum_{j=0}^{s-1} x^{j} P_{0}\left(\sum_{k=0}^{r+s} x^{-k} \alpha_{j+1, k+1}(C(x)-\lambda)\right) u(x) \\
-\lambda \sum_{j=0}^{s-1} x^{j} P_{0}\left(\sum_{k=0}^{r+s} x^{-k} \alpha_{j+1, k+1} u(x)\right) .
\end{gathered}
$$

Let $u=(T-\lambda)^{-1} P_{S} f$. The first term above becomes

$$
\begin{aligned}
-\sum_{j=0}^{s-1} x^{j} P_{0}\left(\sum_{k=0}^{r+s} x^{-k} \alpha_{j+1, k+1}\right) \sum_{\nu=0}^{s-1} g_{v} x^{v} & =-\sum_{j=0}^{s-1} x^{j} P_{0} \sum_{v=0}^{s-1} \sum_{k=0}^{s-1} x^{\nu-k} \delta(j-k) g_{v} \\
& =-\sum_{j=0}^{s-1} x^{j} g_{j}=-P_{S} f .
\end{aligned}
$$

Thus, the total is merely

$$
\begin{aligned}
-\lambda \sum_{j=0}^{s-1} x^{j} P_{0}\left(\sum_{k=0}^{r+s} x^{-k} \alpha_{j+1, k+1}\right) & (T-\lambda)^{-1} \sum_{\nu=0}^{s-1} g_{v} x^{\nu} \\
= & -\lambda \sum_{j=0}^{s-1} x^{j} P_{0}\left(\sum_{k=0}^{r+s} x^{-k} \alpha_{j+1, k+1}\right) A_{+}^{-1} P_{+} A_{-}^{-1} \sum_{\nu=0}^{s-1} g_{v} x^{\nu},
\end{aligned}
$$

where we have used the expression for $(T-\lambda)^{-1}$ which we derived in the last lemma. It is clear that $P_{+} A_{-}^{-1}$ is an analytic projection times an anti-analytic map and hence is represented by an upper triangular matrix. In fact

$$
P_{+} A_{-1}^{-1}\left(\sum_{\nu=0}^{s-1} g_{\nu} x^{\nu}\right)=\sum_{\mu=0}^{s-1} x^{\mu} \sum_{\nu=\mu}^{s-1} A_{-(\nu-\mu)}^{(-1)} g_{\nu},
$$

and thus the matrix of the transformation is

$$
H(\lambda)=\left[\begin{array}{cccc}
{\left[A_{-(0)}^{(-1)}\right]} & {\left[A_{-(1)}^{(-1)}\right]} & \cdots & {\left[A_{-(s-1)}^{(-1)}\right]} \\
{[0]} & {\left[A_{-(0)}^{(-1)}\right]} & & \\
{[0]} & {[0]} & & {\left[A_{-(0)}^{(-1)}\right]}
\end{array}\right]_{(s p) \times(s p)}
$$

which is upper triangular. Also $A_{-(0)}^{(-1)}=A_{-(0)}^{-1}=I$ so $|H(\lambda)| \equiv 1$. 
For arbitrary vectors of the form $\sum_{j=0}^{s-1} h_{j} x^{j}$ we have:

$$
\begin{aligned}
-\lambda \sum_{j=0}^{s-1} x^{j} & P_{0}\left(\sum_{k=0}^{r+s} x^{-k} \alpha_{j+1, k+1}\right) A_{+}^{-1} \sum_{t=0}^{s-1} h_{t} x^{t} \\
& =-\lambda \sum_{j=0}^{s-1} x^{j}\left(\sum_{k=0}^{r+s} \alpha_{j+1, k+1} \sum_{t=0}^{\min (k, s-1)} A_{+(k-t)}^{(-1)} h_{t}\right) \\
& =-\lambda \sum_{j=0}^{s-1} x^{j} \sum_{t=0}^{s-1}\left(\sum_{k=t}^{r+s} \alpha_{j+1, k+1} A_{-(k-t)}^{(-1)}\right) h_{t},
\end{aligned}
$$

and the matrix of this transformation is thus $-\lambda \hat{\alpha} \mathscr{A}(\lambda)=-F(\lambda) \lambda$. The total matrix is $-\lambda \hat{\alpha} \mathscr{A}(\lambda) H(\lambda)$. We have thus shown that $D(\lambda) P_{s}=-\lambda F(\lambda) H(\lambda) P_{s}$. We have in fact shown that

$$
Q\left[I+S(T-\lambda)^{-1}\right] Q=-\lambda F(\lambda) H(\lambda) Q
$$

where $Q \sum_{j=0}^{\infty} f_{j} x^{j}=\sum_{j=0}^{s-1} f_{j} x^{j}$. Now

$$
\left|Q\left(I+S\left(T-\lambda_{0}\right)^{-1}\right) Q\right|=0
$$

iff $\exists Q u \neq 0$ such that $Q u=-S\left(T-\lambda_{0}\right)^{-1} Q u$. Hence $Q u$ is in the range of $S$, or $Q u=P_{S} u$ since $Q \geqq P_{S}$. It is now clear that

$$
\left|Q\left(I+S\left(T-\lambda_{0}\right)^{-1}\right) Q\right|=0 \Leftrightarrow\left|D\left(\lambda_{0}\right)\right|=0
$$

and hence $\left|D\left(\lambda_{0}\right)\right|=0 \Leftrightarrow\left|F\left(\lambda_{0}\right)\right|=0$. Q.E.D.

6. Sufficient conditions for stability (main theorems). We have thus obtained the resolvent $(T+S-\lambda)^{-1}$ if $|\lambda|>1$. We shall now state and prove two important technical lemmas which are concerned with the analyticity in $\lambda$ of each of the factors $A_{+}(x, \lambda)$ and $A_{-}(x, \lambda)$. Notice that each element in $A_{+}(x, \lambda)$ and $A_{-}(x, \lambda)$ is essentially a symmetric function of the roots of a polynomial. Moreover, $\lambda$ appears as a coefficient in this polynomial. We thus expect each factor to be an analytic function of $\lambda$.

The important condition in the next lemma is hypothesis (1). This condition will be called "separation of the zeros". For $|\lambda|>1$, each of the solutions to $p(x)-\lambda=0$ lies either well inside the unit circle $\left(\left|y_{i}(\lambda)\right|<1\right)$ or well outside the unit circle $\left(\left|x_{j}(\lambda)\right|>1\right)$. As $\lambda \rightarrow \lambda_{0}$ with $\left|\lambda_{0}\right|=1$, some of the zeros may approach the unit circle, e.g. $\lim _{\lambda \rightarrow \lambda_{0}} p\left(x_{j}(\lambda)\right)=\lambda_{0}$ for $\left|\lambda_{0}\right|=\left|x_{j}\left(\lambda_{0}\right)\right|=1$. Hypothesis (1) guarantees that $\exists$ no $\lambda_{0}$ on the unit circle such that, for some two zeros $x_{j}(\lambda)$ and $y_{i}(\lambda), x_{j}(\lambda)$ $\rightarrow y_{i}(\lambda)$ as $\lambda \rightarrow \lambda_{0}$, with $|\lambda|>1$.

XVII. Definition And Lemma. Let $p(x)=p_{-s} x^{s}+\cdots+p_{l} / x^{l}$ be as in XIV. Let $|\lambda|>1$. Let

$$
\begin{aligned}
& m_{+}(x, \lambda)=q(\lambda) \prod_{i=1}^{s}\left(x-x_{i}(\lambda)\right), \\
& m_{-}(x, \lambda)=\prod_{i=1}^{l}\left(1-\frac{y_{i}(\lambda)}{x}\right)
\end{aligned}
$$


be the factorization obtained in XIV. We say $p(x)-\lambda$ separates its zeros if there exists a positive quantity $\delta$ such that

$$
\left|x_{i}(\lambda)-y_{j}(\lambda)\right|>\delta
$$

for all $|\lambda|>1$ and all $i, j$.

We now assume

(1) $p(x)-\lambda$ separates its zeros.

(2) $p(x)$ is not identically equal to a constant of absolute value one.

$$
p(x) \not \equiv p_{0} \quad \text { where }\left|p_{0}\right|=1 .
$$

Then the following is true concerning the factors $m_{+}$and $m_{-}$:

$\left(1^{\prime}\right) m_{+}(x, \lambda) / \lambda$ is an analytic function of $x$ and $\lambda$ for all $x \neq \infty$ and $1-d<|\lambda| \leqq \infty$, $d>0$, and

$$
\lim _{\lambda \rightarrow \infty} \frac{m_{+}(x, \lambda)}{\lambda} \equiv-1
$$

$m_{-}(x, \lambda)$ is an analytic function of $x$ and $\lambda$ for $x \neq 0$ and $1-d<|\lambda| \leqq \infty$, and $\lim _{\lambda \rightarrow \infty} m_{-}(x, \lambda) \equiv 1$.

(2') $\lambda m_{+}^{-1}(x, \lambda)$ is analytic if $1-d<|\lambda| \leqq \infty$ and $|x|<1-d^{\prime}, d^{\prime}>0$.

$m_{-1}^{-1}(x, \lambda)$ is analytic if $1-d<|\lambda| \leqq \infty$ and $|x|>1+d^{\prime}$.

Proof. The terms $1-y_{i}(\lambda) / x$ and $x_{j}(\lambda)-x$ are analytic in $\lambda$ for $1 \leqq|\lambda|<\infty$ except perhaps for isolated algebraic branch points. Hence, if the functions

$$
\prod_{i=1}^{l}\left(1-\frac{y_{i}(\lambda)}{x}\right) \text { and } \prod_{j=1}^{s}\left(x-x_{j}(\lambda)\right)
$$

are single valued in $\lambda$ for $\infty>|\lambda| \geqq 1$, then they are both analytic in $\lambda$ for $\infty>|\lambda| \geqq 1$. Consider a point $\lambda_{0}$ with $\infty>\left|\lambda_{0}\right| \geqq 1$. By hypothesis (1) we may take a circle $\Gamma$ in the $\lambda$ plane with center $\lambda_{0}$ and a sufficiently small radius so that

$$
\left|x_{i}(\lambda)-y_{j}(\lambda)\right|>\delta / 2
$$

if $\lambda$ lies in this circle. The factor $m_{-}(x, \lambda)$, for fixed $x$, is a symmetric function of the roots $y_{1}(\lambda) \cdots y_{l}(\lambda)$. Consider any smooth closed path in the $\lambda$ plane which lies entirely within $\Gamma$. We choose any point $\tilde{\lambda}$ in this path and follow the value of $y_{i}(\lambda)$ for each $i$ around the path and back to $\tilde{\lambda}$. Because of (6.1), it is clear that the values of the $y_{i}(\tilde{\lambda}), i=1,2, \ldots, l$, will be at worst permuted among themselves. Thus, by the symmetry of $m_{-}(x, \lambda)$, it follows that $m_{-}(x, \lambda)$ is single valued in this neighborhood of $\lambda_{0}$. The same reasoning works for $\prod_{j=1}^{s}\left(x-x_{j}(\lambda)\right)$. Of course $d(\lambda)$ is analytic in $\lambda$, and hence $m_{+}(x, \lambda)$ is also analytic in this neighborhood of $\lambda_{0}$.

Now we examine the behavior of the factors near $\lambda=\infty$. If $l \leqq 0$, then $m_{-}(x, \lambda) \equiv 1$. If $l>0$, then $\forall \varepsilon>0$, consider the circle $|x| \equiv \varepsilon$. We may choose $\tilde{\lambda}(\varepsilon)$ so that

$$
|\tilde{\lambda}(\varepsilon)|>\sup _{-\pi<\theta \leqq \pi}\left|p\left(\varepsilon e^{i \theta}\right)\right| \text {. }
$$


Then $p(x)-\tilde{\lambda}(\varepsilon)$ has index zero on the circle $|x|=\varepsilon$. Thus

$$
\left|y_{i}(\tilde{\lambda}(\varepsilon))\right|<\varepsilon, \quad i=1,2, \ldots, l .
$$

Hence $\lim _{\lambda \rightarrow \infty} m_{-}(x, \lambda) \equiv 1$ and we may show that $m_{-}(x, \lambda)$ is analytic and single valued in $\lambda$ near $\lambda=\infty$, using the same reasoning as we used in the last paragraph about permuting the $y_{i}(\lambda)$. If $s=0$, then $m_{+}(x, \lambda) \equiv-\lambda+p_{0}$. If $s<0$, then $m_{+}(x, \lambda)$ $\equiv-\lambda$. If $s>0$, then $\forall \varepsilon>0$ we may take

$$
|\widetilde{\lambda}(1 / \varepsilon)|>\sup _{-\pi<\theta \leqq \pi}\left|p\left((1 / \varepsilon) \cdot e^{i \theta}\right)\right| .
$$

Then $p(x)-\widetilde{\pi}(1 / \varepsilon)$ has index zero on the circle $|x|=1 / \varepsilon$ and hence

$$
\left|x_{j}(\widetilde{\lambda}(1 / \varepsilon))\right|>1 / \varepsilon, \quad j=1,2, \ldots, s .
$$

Thus $m_{+}(x, \lambda)$ is single valued near $\lambda=\infty$ and has a pole at $\lambda=\infty$. Hence we now have:

$$
\begin{aligned}
& m_{+}(x, \lambda)=d_{j}(x) \lambda^{j}+d_{j-1}(x) \lambda^{j-1}+\cdots \\
& m_{-}(x, \lambda)=1+\frac{b_{-1}(x)}{\lambda}+\cdots
\end{aligned}
$$

If we match coefficients of $\lambda$ in the equation $m_{-} m_{+}=p(x)-\lambda$ we obtain $j=1$ and $d_{j}(x) \equiv-1$. We thus have proven conclusion $\left(1^{\prime}\right)$. Conclusion $\left(2^{\prime}\right)$ would follow immediately from the analyticity of $m_{+}(x, \lambda) / \lambda$ and $m_{-}(x, \lambda)$ if we knew that each factor was nonsingular in the regions described in $\left(2^{\prime}\right)$. It is clear that

$$
\prod_{i=1}^{l}\left(1-\frac{y_{i}(\lambda)}{x}\right) \text { and } \prod_{j=1}^{s}\left(x-x_{j}(\lambda)\right)
$$

are both nonsingular in the respective regions. The constant $q(\lambda)$ is independent of $\lambda$ unless $s \leqq 0$. If $s<0, q(\lambda)=-\lambda$. If $s=0$, then $q(\lambda)=-\lambda+p_{0}$. By hypothesis (2), if $l=0=s$, then $\left|p_{0}\right|<1$. If $l>0$, then

$$
p(x)-\lambda=\left(-\lambda+p_{0}\right) \prod_{i=1}^{l}\left(1-\frac{y_{2}(\lambda)}{x}\right), \quad\left|y_{i}(\lambda)\right| \leqq 1, i=1,2, \ldots, l \quad|\lambda| \geqq 1 .
$$

and if $\left|p_{0}\right| \geqq 1$, then $p(x) \equiv p_{0}$, which is a contradiction. Thus $q(\lambda)$ is nonsingular in the proper region. The result $\left(2^{\prime}\right)$ is now immediate.

We may now use these results in an obvious way in order to obtain analyticity in $\lambda$ of $A_{+}(x, \lambda), A_{-}(x, \lambda), F(\lambda)$, and their inverses.

XVIII. Lemma. Suppose

$$
\sup _{\nu=1,2, \ldots, p ; 0 \leqq \theta \leqq 2 \pi}\left|c^{(v)}\left(e^{i \theta}\right)\right| \leqq 1
$$

We make the following two assumptions:

(1) For each $\nu$, the zeros of $c^{(v)}(x)-\lambda$ are "separated" in the sense defined in the 
last definition and lemma. That is, there exists a positive quantity $\delta$ such that

$$
\left|x_{i}^{(v)}(\lambda)-y_{j}^{(\nu)}(\lambda)\right|>\delta \text { for all }|\lambda|>1 \text { and all } i, j, \nu .
$$

(2) For each $\nu, c^{(v)}(x)$ is not identically equal to a constant of absolute value one, $c^{(v)}(x) \not \equiv c_{0}^{(v)}$ for $\left|c_{0}^{(v)}\right|=1$.

Then the quantities $A_{+}(x, \lambda), A_{-}(x, \lambda), A_{+}^{-1}(x, \lambda), A_{-}^{-1}(x, \lambda)$ and $F(\lambda)$ (which were defined in $\mathrm{XV}$ and $\mathrm{XVI})$ have the following properties:

$\left(1^{\prime}\right) A_{+}(x, \lambda) I / \lambda$ is an analytic matrix function of $x$ and $\lambda$ for all $x \neq \infty$ and $1-d$ $<|\lambda| \leqq \infty$ for some $d>0$ and

$$
\lim _{\lambda \rightarrow \infty} A_{+}(x, \lambda) I / \lambda \equiv-I \text {. }
$$

$A_{-}(x, \lambda)$ is an analytic matrix function of $x$ and $\lambda$ for all $x \neq 0$ and $1-d<|\lambda| \leqq \infty$ and $\lim _{\lambda \rightarrow \infty} A_{-}(x, \lambda) \equiv I$.

(2') $\lambda I A_{+}^{-1}(x, \lambda)$ is analytic in both variables if $1-d<|\lambda| \leqq \infty$ and $|x|<1-d^{\prime}$, $d^{\prime}>0$.

$A=1(x, \lambda)$ is analytic in both variables if $1-d<|\lambda| \leqq \infty$ and $|x|>1+d^{\prime}$.

(3') $\lambda I F(\lambda)$ is analytic if $\infty \geqq|\lambda|>1-d$ and $\lim _{\lambda \rightarrow \infty} \lambda I F(\lambda)=-I$.

Proof. This is an immediate corollary of the last lemma and of the definitions of these quantities. Q.E.D.

We may now proceed to prove our main theorem. We do this without requiring that 2.4 be consistent with the P.D.E. (2.1). The strategy we shall use is as follows:

(1) We apply Theorem VI to $L=T+S$, with $P$ being the projection onto the first $r+l+s$ components of a vector in $\left(l_{2}\right)_{(p \times 1)}$.

(2) Assumptions (1) and (2) of that theorem are immediately verified since $(I-P) S=S(I-P)=0$ and $\|T\| \leqq 1$.

(3) We use the resolvent (5.1) in Lemma IX in order to verify condition (3). This operator will be used as a generating function:

$$
(T+S-\lambda)^{-1}=-\sum_{n=0}^{\infty} \frac{(T+S)^{n}}{\lambda^{n+1}} \text { for }|\lambda|>1
$$

XIX. MaIn Theorem. Suppose

(1) $\sup _{v=1,2, \ldots, p ;-\pi<\theta \leqq \pi}\left|c^{(v)}\left(e^{i \theta}\right)\right| \leqq 1$.

(2) For each $v$ the zeros of $c^{(v)}(x)-\lambda$ are separated as defined in XVII. That is, there exists a positive quantity $\delta$ such that $\left|x_{i}^{(v)}(\lambda)-y_{j}^{(\nu)}(\lambda)\right|>\delta$ for all $|\lambda|>1$, and all $i, j, \nu$.

(3) For each $\nu, c^{(v)}(x)$ is not identically equal to a constant of absolute value one, $c^{(v)}(x) \not \equiv c_{0}^{(v)}$ with $\left|c_{0}^{(v)}\right|=1$.

(4) $|F(\lambda)| \neq 0$ for $\infty>|\lambda| \geqq 1$. (Recall $|F(\lambda)|=$ determinant of $F(\lambda)$ and $F(\lambda)$ $=\hat{\alpha} \mathscr{A}(\lambda)$.) Then 2.4 is stable.

Proof. We apply Theorem VI to $L=T+S$. Let

$$
P\left(\sum_{j=0}^{\infty} f_{j} x^{j}\right)=\sum_{j=0}^{r+l+s} f_{j} x^{j} .
$$


Then $(I-P) S=S(I-P)=0$. Moreover, hypothesis (1) guarantees that $\|T\| \leqq 1$. Thus conditions (1) and (2) of Theorem VI are verified for $L=T+S$. From conditions (1) and (4) of this theorem, VII, IX, X, and XVI, it follows that $T+S-\lambda$ is invertible for $|\lambda|>1$ and

$$
(T+S-\lambda)^{-1}=(T-\lambda)^{-1}-(T-\lambda)^{-1}\left(I+S(T-\lambda)^{-1}\right)^{-1} S(T-\lambda)^{-1} .
$$

If we can show that $P(T+S-\lambda)^{-1} P$ is a bounded analytic operator for $|\lambda|>$ $1-\delta^{\prime}, \delta^{\prime}>0$, it will then follow by the Cauchy integral formula that

$$
\left\|P(T+S)^{n} P\right\| \leqq k\left(1-\delta^{\prime} / 2\right)^{n}, \quad k>0
$$

and hypothesis (3) of Lemma VI will be verified with

$$
a_{n}=k\left(1-\delta^{\prime} / 2\right)^{n}, \quad a=2 k / \delta^{\prime} .
$$

Thus $(T+S)$ will indeed be power-bounded.

We claim that $\exists$ a finite set of matrices $J_{v t}(\lambda), 0 \leqq \nu, t \leqq r+l+s,|\lambda|>1$ such that

$$
P(T+S-\lambda)^{-1} P u=\sum_{v=0}^{r+s+l} x^{v}\left(\sum_{t=0}^{r+s+l} J_{v t}(\lambda) u_{t}\right)
$$

This is clear because the range of $P$ is just the space spanned by arbitrary vectors of the form

$$
\sum_{j=0}^{r+l} f_{j} x^{j}
$$

We make the following important claim: each matrix $J_{v t}(\lambda)$ is analytic in $\lambda$ for $|\lambda|>1-\delta^{\prime}$ where $1>\delta^{\prime}>0$ and $\delta^{\prime}$ is a constant independent of $\nu$ and $t$. We first calculate $P(T-\lambda)^{-1} P$ using Lemma XV. After some simple calculations we have

$$
P(T-\lambda)^{-1} P u=\sum_{v=0}^{r+s+l} x^{v}\left(\sum_{k=0}^{v} A_{+(v-k)}^{(-1)}(\lambda)\left(\sum_{t=k}^{r+s+l} A_{-(t-k)}^{(-1)}(\lambda) u_{t}\right)\right) .
$$

We next calculate

$$
P(T-\lambda)^{-1}\left(I+S(T-\lambda)^{-1}\right)^{-1} S(T-\lambda)^{-1} P
$$

using Lemmas XV and XVI, and the definition below of the $N_{j+1, k+1}(\lambda)$. After some simpla but lengthier calculations, we arrive at:

$-P(T-\lambda)^{-1}\left(I+S(T-\lambda)^{-1}\right)^{-1} S(T-\lambda)^{-1} P$

$$
=-\sum_{v=0}^{r+l+s} x^{v}\left(\sum_{t=0}^{\min (v, s-1)} A_{+(v-t)}^{(-1)}(\lambda)\right.
$$

$$
\begin{aligned}
\cdot\left(\sum_{k=0}^{s-1} \frac{N_{t+1, k+1}(\lambda)}{\lambda}\right. & \left(\sum _ { \Phi = 0 } ^ { r + s } \alpha _ { k + 1 , \oplus + 1 } \left(\sum_{j=\max (\Phi-s, 0)}^{\phi+l} C_{j-\Phi}\right.\right. \\
& \left.\left.\left.\cdot\left(\sum_{\xi=0}^{j} A_{+(j-\xi)}^{(-1)}(\lambda)\left(\sum_{\mu=\xi}^{r+l+s} A_{-(\mu-\xi)}^{(-1)} u_{\mu}\right)\right)\right)\right)\right)
\end{aligned}
$$


where we have defined the matrices $N_{t+1, j+1}(\lambda)$ for $0 \leqq t, j \leqq s-1$ and $|\lambda|>1$ as follows:

$$
F^{-1}(\lambda)=\left[\begin{array}{ccc}
{\left[N_{11}(\lambda)\right]_{p \times p}} & \cdots & {\left[N_{1 s}\right]_{p \times p}} \\
\vdots & & \\
{\left[N_{s 1}\right]_{p \times p}} & \cdots & {\left[N_{s s}\right]_{p \times p}}
\end{array}\right]_{(s p \times s p)}
$$

Conditions (1), (2), (3) and Lemma XVIII assure us that each $\lambda A_{+(j)}^{(-1)}(\lambda)$ and $A_{-(j)}^{(-1)}(\lambda)$ is analytic in $\lambda$ for $1-d<|\lambda| \leqq \infty$. Condition (4) and the same lemma assure us that each $N_{t+1, k+1}(\lambda) / \lambda$ is analytic in $\lambda$ for $1-d^{\prime \prime}<|\lambda| \leqq \infty, d^{\prime \prime}>0$. Hence, using (5.1), (6.2) and (6.3), each $J_{v t}(\lambda)$ is a finite linear combination of products of matrices which are analytic for $1-\delta^{\prime}<|\lambda| \leqq \infty, 1>\delta^{\prime}>0$. Thus the analyticity of each $J_{v t}(\lambda)$ follows.

Stability is now immediate. Q.E.D.

We shall now compare our results with those of H.-O. Kreiss [1]. In fact we shall obtain his main result for the constant coefficient case as a corollary to our main theorem. We first make his assumptions. In particular, we notice that he assumes consistency with the partial differential equation and boundary conditions (2.1), (2.2) and (2.3). Moreover, he needs the restriction of dissipativity. We have essentially replaced these conditions by requiring that $c^{(v)}(x)$ not be a constant of absolute value one and by our condition of "separation of zeros", or "positive distance between zeros". We shall show that our conditions are more general. We shall also give a nondissipative example for which stability may be verified by using our main theorem.

Assumptions. (1) The difference equations (2.4a) and (2.4b) are consistent with the hyperbolic system of partial differential equations (2.1), (2.2) and (2.3).

(2) $\exists$ no nontrivial solution to (2.4) of the form

$$
v_{j}^{n}=\lambda_{0}^{n} \psi_{j} \text { for }\left|\lambda_{0}\right|>1 \text { and } \sum_{j=0}^{\infty}\left|\psi_{j}\right|^{2}<\infty \text {. }
$$

(3) The difference approximation is dissipative, i.e. $\exists \delta>0$ and a natural number $t>0$ so that

$$
\left|c^{(v)}\left(e^{i \theta}\right)\right| \leqq 1-\delta|\theta|^{2 t} \quad \text { for } \nu=1,2, \ldots, p .
$$

XX. LEMmA (KREISS). If assumptions (1), (2) and (3) are valid, it then follows that $\exists \varepsilon$ with $0<\varepsilon<1 \ni$ for any $\lambda \geqslant|\lambda|>1$ :

(a) For $1 \leqq j \leqq n_{0}, \exists$ no $y_{i}^{(j)}(\lambda)$ with $1-\varepsilon \leqq\left|y_{i}^{(j)}(\lambda)\right| \leqq 1$.

(b) For $n_{0}+1 \leqq j \leqq p, \exists$ no $x_{i}^{(j)}(\lambda)$, with $1 \leqq\left|x_{i}^{(j)}(\lambda)\right| \leqq 1+\varepsilon$.

(c) If $|\lambda|=1$ and $\lambda \neq 1$ then $\exists$ no $x_{i}^{(j)}(\lambda)$ or $y_{i}^{(j)}(\lambda)$ of absolute value one. For $j=1,2, \ldots, n_{0}$, there exists exactly one $i(j)$ such that $\left|x_{i(j)}^{(j)}(1)\right|=1$, in fact $x_{i(j)}^{(j)}(1)=1$.

For $j=n_{0}+1, \ldots, p$, there exists exactly one $i(j)$ such that $\left|y_{i(j)}^{(j)}(1)\right|=1$, in fact $y_{i(j)}^{(j)}(1)=1$.

(d) $c^{(v)}(x)$ is not a constant for each $v$. 
These properties clearly imply that the zeros are separated.

Proof. See Kreiss [1, Lemmas 6 and 7].

XXI. Lemma. Suppose assumptions (1), (2) and (3) are valid. In addition, equation (2.4) has no nontrivial solution of the form $v_{j}^{n}=\lambda^{n} \Psi_{j}$ with $|\lambda|=1$ and

(a) $\sum_{j=0}^{\infty}\left|\Psi_{j}\right|^{2}<\infty$ if $\lambda \neq 1$.

(b) $\left|\Psi_{j}^{I}\right| \leqq k,\left|\Psi_{j}^{I I}\right| \leqq k \mu^{j}$, if $\lambda=1 ; j=0,1, \ldots$

with $0 \leqq \mu<1$ and $k>0$. It then follows that $|F(\lambda)| \neq 0$ for any $\lambda$ with $|\lambda|=1$.

Proof. If $\left|F\left(\lambda_{0}\right)\right|=0$, then $\exists$ a nontrivial eigenvector $g$ with $F(\lambda) g=0$. Let $h_{j}$, $j=0,1, \ldots, s-1$, be defined by

$$
g=\left[\left[h_{0}\right]_{(p \times 1)}^{T}\left[h_{1}\right]_{(p \times 1)}^{T} \cdots\left[h_{(s-1)}\right]_{(p \times 1)}^{T}\right]_{(s p \times 1)}^{T} .
$$

Let

$$
\Psi_{j}=\sum_{k=0}^{\min (j . s-1)} A_{+(j-k)}^{(-1)}(\lambda) h_{k} .
$$

It then follows by using the definition of $F\left(\lambda_{0}\right)$ that

$$
\sum_{\mu=0}^{r+s} \alpha_{j+1, \mu+1} \Psi_{\mu} \equiv 0, \quad j=0,1, \ldots, s-1
$$

We claim

$$
\sum_{j=0}^{\infty} \Psi_{j} x^{j}=A_{+}^{-1}\left(x, \lambda_{0}\right) \sum_{k=0}^{s-1} h_{k} x^{k}
$$

is a nontrivial solution to $(T+S) \Psi(x)=\lambda_{0} \Psi(x)$ but $\Psi(x)$ may not be an element of $\left(\hat{l}_{2}\right)_{(p \times 1)}$. This is true because the general solution to the problem

$$
\sum_{k=-s}^{l} C_{k} u_{j+k}=\lambda u_{j}, \quad j=s, s+1, \ldots
$$

for $|\lambda|>1$, in $\left(l_{2}\right)_{(p \times 1)}$ is a linear combination of the solutions to

$$
P_{+} x^{-s}[C(x)-\lambda] \Psi(x)=0 .
$$

Let $\Psi(x)=\sum_{j=0}^{s-1} x^{j} A_{+}^{-1}(x, \lambda) h_{j}$, then

$$
P_{+} x^{-s}[C(x)-\lambda] x^{j} A_{+}^{-1}(x, \lambda)=P_{+} x^{j-s} A_{-}^{-1}(x, \lambda)=0 \text { if } j<s .
$$

We now let $\lambda \rightarrow \lambda_{0}$. We already know that

$$
\sum_{\mu=0}^{r+s} \alpha_{j+1, \mu+1} \Psi_{\mu}=0, \quad j=0,1, \ldots, s-1
$$

for $\lambda=\lambda_{0}$. Because of the continuity of each $A_{j}^{(-1)}(\lambda)$ for $|\lambda|>1-d$, it is clear that

$$
\sum_{k=-s}^{l} C_{k} \Psi_{j+k} \rightarrow \lambda_{0} \Psi_{j} \quad \text { as } \lambda \rightarrow \lambda_{0}, \quad j=s, s+1, \ldots
$$

The claim is thus proven. 
We shall now estimate the coefficients

$$
A_{+j}^{(-1)}\left(\lambda_{0}\right), \quad j=0,1, \ldots
$$

If $\lambda_{0} \neq 1$, then by conclusion (c) of the last lemma, $\exists \mu^{\prime}\left(\lambda_{0}\right)$ with $0<\mu^{\prime}\left(\lambda_{0}\right)<1$ such that

$$
\left|1 / x_{i}^{(j)}\left(\lambda_{0}\right)\right| \leqq \mu^{\prime}\left(\lambda_{0}\right) \text { for all } i, j
$$

This implies that $\Psi(x)$ is in $\left(\hat{l}_{2}\right)_{(p \times 1)}$. If $\lambda_{0}=1$, then $\exists \mu^{\prime}$ with $0<\mu^{\prime}<1$ such that $\left|1 / x_{i}^{(j)}(1)\right| \leqq \mu^{\prime}$ for all $i$ if $j=n_{0}+1, n_{0}+2, \ldots, p$; and for all $i$ but one exceptional $i(j)$ for each $j=1,2, \ldots, n_{0}$, and $x_{i(j)}^{(j)}(1)=1$ for this $i(j)$. It then follows that

$$
\left|\Psi_{j}^{I}\right| \leqq k, \quad\left|\Psi_{j}^{I I}\right| \leqq k \mu^{j}, \quad j=0,1, \ldots
$$

for $k>0$ and $0<\mu<1$.

Thus $v_{j}^{n}=\lambda_{0}^{n} \Psi_{j}$ has the properties forbidden in the hypothesis and hence $|F(\lambda)|$ $\neq 0$ if $|\lambda|=1$. Q.E.D.

XXII. Theorem (Kreiss). Suppose assumptions (1), (2), and (3) are valid. Assume equation (2.4) has no nontrivial solution of the form $v_{j}^{n}=\lambda^{n} \Phi_{j}$ with $|\lambda|=1$ and

(a) $\sum_{j=0}^{\infty}\left|\Phi_{j}\right|^{2}<\infty$ if $\lambda \neq 1$.

(b) $\left|\Phi_{j}^{I}\right|<k,\left|\Phi_{j}^{I I}\right| \leqq k \mu^{j}$ if $\lambda=1$ and $j=0,1, \ldots$ with $0 \leqq \mu<1$ and $k>0$.

Then (2.4) is stable.

Proof. We must merely verify the conditions of Theorem XIX. This verification is immediate from the assumptions, the last two lemmas, Lemma $\mathrm{X}$, and Lemma XVI.

XXIII. EXample. Consider the hyperbolic P.D.E. in the quarter-plane $u_{t}=u_{x}, x, t \geqq 0 u(x, 0)=\Phi(x)$. Take the difference approximation

$$
\begin{aligned}
u_{j}^{n+1} & =\frac{1+\mu}{2} u_{j+1}^{n}+\frac{1-\mu}{2} u_{j-1}^{n}, \quad j=1,2, \ldots, n=0,1, \ldots \\
u & =\frac{\Delta t}{\Delta x}, \quad 0<\mu<1 \\
u_{0}^{n+1} & =2 u_{1}^{n+1}-u_{2}^{n+1}, \quad n=0,1,2, \ldots, \\
\frac{u_{j}^{n+1}-u_{j}^{n}}{\Delta t} & =\left(\frac{1}{2 \mu}+\frac{1}{2}\right)\left[\frac{u_{j+1}^{n}-u_{j}^{n}}{\Delta x}\right]+\left(\frac{1}{2}-\frac{1}{2 \mu}\right)\left[\frac{u_{j}^{n}-u_{j-1}^{n}}{\Delta x}\right] \\
& =u_{x}(j \Delta x, n \Delta t)+O(\Delta t), u_{0}^{n+1}-2 u_{1}^{n+1}+u_{2}^{n+1}=O\left((\Delta t)^{2}\right) .
\end{aligned}
$$

Thus we have consistency.

$$
\begin{aligned}
C\left(e^{i \theta}\right) & =\cos \theta-\mu i \sin \theta \\
\left|C\left(e^{i \theta}\right)\right| & =\left[1+\left(\mu^{2}-1\right) \sin ^{2} \theta\right]^{1 / 2} \leqq 1 \quad \text { if } 0<\mu<1 . \\
\left|C\left(e^{i \pi}\right)\right| & =1
\end{aligned}
$$


Thus the approximation is not dissipative, so we cannot use Theorem XXII. However

$$
\begin{aligned}
C(x)-\lambda & =\frac{1-\mu}{2}\left(x-x_{1}(\lambda)\right)\left(1-\frac{y_{1}(\lambda)}{x}\right) \\
& =\left(\frac{1+\mu}{2}\right) 1 / x-\lambda+\left(\frac{1-\mu}{2}\right) x .
\end{aligned}
$$

Hence

$$
\left|x_{1}(\lambda) y_{1}(\lambda)\right|=\left(\frac{1+\mu}{1-\mu}\right)>1 \text { or }\left|x_{1}(\lambda)\right|>1 /\left|y_{1}(\lambda)\right|
$$

for $|\lambda| \geqq 1$. Thus the zeros are separated. Moreover,

hence

$$
A_{+}^{-1}(x, \lambda)=-2 / 1-\mu \sum_{j=0}^{\infty} \frac{x^{j}}{\left(x_{1}(\lambda)\right)^{j+1}}
$$

$$
F(\lambda)=-2 /(1-\mu) x_{1}(\lambda)\left[1-2 / x_{1}(\lambda)+1 / x_{1}^{2}(\lambda)\right] .
$$

If $F\left(\lambda_{0}\right)=0$ for $\infty>\left|\lambda_{0}\right| \geqq 1$, then $x_{1}\left(\lambda_{0}\right)=1$. This is a contradiction.

We thus have stability. Q.E.D.

7. Appendix-Implicit difference equations. The apparatus we have set up in the previous sections in order to handle the stability theory of explicit difference schemes may be modified to include implicit difference approximations to (2.1). See [13] for a thorough description of the implicit case. In this appendix we shall discuss some of the highlights of the stability theory of implicit difference equations.

The difference approximation is of the form

(7.1)(a) $\sum_{k=-s}^{l} B_{k} v_{k+j}^{n+j}=\sum_{k=-s}^{l} C_{k} v_{k+j}^{n}, \quad j=s, s+1, \ldots, n=0,1, \ldots$,

with boundary conditions

(7.1)(b) $\sum_{k=0}^{r+s} \alpha_{j+1, k+1} v_{k}^{n+1}=\sum_{k=0}^{r+s} \gamma_{j+1, k+1} v_{k}^{n}, \quad j=0,1, \ldots, t-1, n=0,1, \ldots$, where $s>0$ and $\left[C_{-s}\right] \neq[0]$.

The $B_{k}$ and $C_{k}$ are again diagonal $(p \times p)$ matrices, $\alpha_{j k}$ and $\gamma_{j k}$ are again $(p \times p)$ matrices,

$$
B(x)=\sum_{k=-s}^{l} B_{k} x^{-k}=\left[\begin{array}{ccc}
b^{(1)}(x) & & 0 \\
& \ddots & \\
0 & & b^{(p)}(x)
\end{array}\right]
$$

and

$$
C(x)=\sum_{k=-s}^{l} C_{k} x^{-k}=\left[\begin{array}{ccc}
c^{(1)}(x) & & 0 \\
& \ddots & \\
0 & & c^{(p)}(x)
\end{array}\right]
$$


Moreover, we assume that each $b^{(j)}(x)$ has as many zeros inside the unit circle counting multiplicity as it has poles at $x=0$. (See [13] for an explanation of this last assumption.)

We notice that if (1) $t=s$, (2) $\gamma_{j k} \equiv 0$, (3) $B_{k}=\delta(k) I$, (4) $\alpha_{j k}=\delta_{(j-k)} I$ for $0 \leqq j$, $k \leqq s-1$; then this problem reduces to the explicit case.

XXIV. RemarK. Equation (7.1) may be rewritten on $\left(\hat{l}_{2}\right)_{(p \times 1)}$ as

$$
\left(T_{0}+S_{0}\right) v^{n+1}(x)=\left(T_{1}+S_{1}\right) v^{n}(x) .
$$

$T_{0}$ and $T_{1}$ are again Toeplitz matrices; $S_{0}$ and $S_{1}$ are again finite-dimensional operators:

$$
\begin{aligned}
T_{0} v^{n+1} & =P_{+} x^{t-s} B(x) v^{n+1}(x) \\
T_{1} v^{n} & =P_{+} x^{t-s} C(x) v^{n}(x) \\
S_{0} v^{n+1} & =\sum_{j=0}^{t-1} x^{j} P_{0}\left[-x^{-j+t-s} B(x)+\sum_{k=0}^{r+s} \alpha_{j+1, k+1} x^{-k}\right] v^{n+1}(x), \\
S_{1} v^{n} & =\sum_{j=0}^{t-1} x^{j} P_{0}\left[-x^{-j+t-s} C(x)+\sum_{k=0}^{r+s} \gamma_{j+1, k+1} x^{-k}\right] v^{n}(x) .
\end{aligned}
$$

We thus wish to obtain

(a) necessary and sufficient conditions for $\left(T_{0}+S_{0}\right)^{-1}$ to exist, and

(b) sufficient conditions so that $\left\|\left[\left(T_{0}+S_{0}\right)^{-1}\left(T_{1}+S_{1}\right)\right]^{n}\right\| \leqq M$ for all $n=0,1, \ldots$

XXV. THEOREM. The following conditions are necessary and sufficient for the operator $T_{0}+S_{0}$ to be invertible on $\left(\hat{l}_{2}\right)_{(p \times 1)}$ :

(1) $b^{(v)}\left(e^{i \theta}\right) \neq 0$ for $\nu=1,2, \ldots, p ; 0 \leqq \theta \leqq 2 \pi$,

(2) $t=s$,

(3) $\left(T_{0}+S_{0}\right) v=0 \Leftrightarrow v=0$.

We now assume throughout that $\left(T_{0}+S_{0}\right)^{-1}$ exists.

XXVI. RemarK. Condition (a) $\Rightarrow$ (b) $\Leftrightarrow$ (c); but (c), in general, does not imply (a).

(a) $\left(T_{0}+S_{0}\right)^{-1}\left(T_{1}+S_{1}\right)$ is power bounded.

(b) $\left|\sigma\left(\left(T_{0}+S_{0}\right)^{-1}\left(T_{1}+S_{1}\right)\right)\right| \leqq 1$.

(c) $\sup _{v=1,2, \ldots, p ; 0 \leqq \theta \leqq 2 \pi}\left|q^{(v)}\left(e^{i \theta}\right)\right| \leqq 1$ where $q^{(v)}(x)=c^{(v)}(x) / b^{(v)}(x)$

and $\left(T_{1}+S_{1}-\lambda\left(T_{0}+S_{0}\right)\right) v=0$ for $|\lambda|>1 \Leftrightarrow v=0$.

Moreover, condition (b) implies that $\exists k_{1}, k_{2}>0$ such that

$$
\left\|\left[\left(T_{0}+S_{0}\right)^{-1}\left(T_{1}+S_{1}\right)\right]^{n}\right\| \leqq k_{1} n^{k_{2}}
$$

XXVII. Definition. We may use the results of XII in much the same way as in $\mathrm{XV}$ to factor

$$
C(x)-\lambda B(x)=A_{-}(x, \lambda) A_{+}(x, \lambda) \text { for }|\lambda|>1
$$


such that

$$
\left(T_{1}-\lambda T_{0}\right)^{-1} u=A_{+}^{-1}(x, \lambda) P_{+} A_{-}^{-1}(x, \lambda) u
$$

We may then define the matrix $\mathscr{A}(\lambda)$ as in XVI. Let

$$
F(\lambda)=\frac{[\hat{\gamma}-\lambda \hat{\alpha}] \mathscr{A}(\lambda)}{-\lambda}
$$

where

$$
\hat{\gamma}-\lambda \hat{\mathscr{A}}=\left[\begin{array}{ccc}
{\left[\gamma_{11}-\lambda \alpha_{11}\right]\left[\gamma_{12}-\lambda \alpha_{12}\right]} & \cdots & {\left[\gamma_{1, r+s+1}-\lambda \alpha_{1, r+s+1}\right]} \\
{\left[\gamma_{21}-\lambda \alpha_{21}\right]\left[\gamma_{22}-\lambda \alpha_{22}\right]} & \cdots & {\left[\gamma_{2, r+s+1}-\lambda \alpha_{2, r+s+1}\right]} \\
\vdots & & \\
{\left[\gamma_{s 1}-\lambda \alpha_{s 1}\right]\left[\gamma_{s 2}-\lambda \alpha_{s 2}\right]} & \cdots & {\left[\gamma_{s, r+s+1}-\lambda \alpha_{s, r+s+1}\right]}
\end{array}\right] .
$$

XXVIII. MaIn Theorem (Implicit CASE). The following conditions are sufficient for stability:

(1) $\sup _{v=1,2, \ldots, p ; 0 \leqq \theta \leqq 2 \pi}\left|q^{(v)}\left(e^{i \theta}\right)\right| \leqq 1$.

(2) For each $\nu, c^{(v)}(x)-\lambda b^{(v)}(x)$ separates its zeros in the same way as $p(x)-\lambda$ did in XVII.

(3) For each $\nu, c^{(v)}(x) / b^{(v)}(x) \not \equiv p_{0}^{(v)}$, where $\left|p_{0}^{(v)}\right|=1$.

(4) $|F(\lambda)| \neq 0$ for $\infty>|\lambda| \geqq 1$.

XXIX. EXAmple. Approximate the scalar hyperbolic P.D.E. in the quarter plane $x, t \geqq 0$ :

$$
u_{i}=u_{x} \quad \text { with } u(x, 0)=\Phi(x)
$$

by the implicit difference scheme

$$
\begin{gathered}
u_{j}^{n+1}-\mu / 2 u_{j+1}^{n+1}+\mu / 2 u_{j-1}^{n+1}=\frac{u_{j+1}^{n}+u_{j-1}^{n}}{2}, \quad j=1,2, \ldots, n=0,1, \ldots, \\
\left(u=\frac{\Delta t}{\Delta x}, 0<\mu\right),
\end{gathered}
$$

and boundary conditions

$$
u_{0}^{n+1}=2 u_{1}^{n+1}-u_{2}^{n+1}, \quad n=0,1, \ldots
$$

Consistency, invertibility, and stability may be easily verified for this nondissipative implicit example.

The author would like to thank Dr. A. Chorin for suggesting the idea upon which this work is based and for participating in many interesting discussions relating to it; Professor H.-O. Kreiss for asking incisive questions; Professor G. Strang for valuable discussions on the implicit case and related topics; and Professor P. D. Lax for graciously giving much time and patience in directing the research and for contributing many ideas to the final result. 


\section{BIBLIOGRAPHY}

1. Heinz-Otto Kreiss, "Difference approximations for the initial-boundary value problem for hyperbolic differential equations," in Numerical solutions of nonlinear partial differential equations, Edited by D. Greenspan, Wiley, New York, 1966, pp. 141-166.

2. G. Strang, Wiener-Hopf difference equations, J. Math. Mech. 13 (1964), 85-96.

3. - Implicit difference methods for initial-boundary value problems, J. Math. Anal. Appl. 16 (1966), 188-198.

4. I. C. Gohberg and M. G. Krein, Systems of integral equations on a half line with kernels depending on the difference of arguments, Amer. Math. Soc. Transl. 14 (1960), 217-288.

5. R. D. Richtmyer, The stability criterion of Godunov and Ryabenkii for difference schemes, A.E.C. Research and Development Report NYO-1480-4, 1964.

6. Peter D. Lax and Burton Wendroff, On the stability of difference schemes, Comm. Pure Appl. Math. 15 (1962), 363-371.

7. R. D. Richtmyer, Difference methods for initial value problems, Interscience, New York, 1957.

8. S. K. Godunov and V. S. Ryabenkiī, Spectral criteria for the stability of boundary-value problems for non-selfadjoint difference equations, Uspehi Mat. Nauk 18 (1963), no. 3(111), 3-15.

9. - Canonical forms of systems of linear ordinary difference equations with constant coefficients, Ž. Vyčisl. Mat. i Mat. Fiz. 3 (1963), 211-222.

10. —, Theory of difference schemes, an introduction, North-Holland, Amsterdam, 1964.

11. N. Dunford and J. T. Schwartz, Linear operators, Part 1, Interscience, New York, 1958.

12. S. J. Osher, Systems of difference equations with general homogeneous boundary conditions, Brookhaven National Laboratory Report, BNL 11246, 1967.

13. — Stability of mixed implicit difference schemes, Brookhaven National Laboratory Report, BNL 11784, 1967.

Brookhaven NATIONAL LABORATORY, UPTON, L. I., NEW YoRK 